\title{
Can a multi-factorial assessment and interventional programme decrease inpatient falls in an elderly care ward?
}

Rebecca SJ Gibson, April Heaney, Karen Hull

\begin{abstract}
Each year approximately 282,000 inpatient falls are reported to the National Patient Safety Agency (NPSA). A significant number result in death, or moderate to severe injury. (1) Research shows that falls may be reduced by 18 to $31 \%$ through multi-factorial assessments and interventions. (4) If a fall cannot be prevented, the patient should receive a prompt and effective response to achieve the best possible recovery and avoidance of further falls.
\end{abstract}

Using 'Plan-Do-Study-Act' learning cycles, our aims were to decrease the inpatient falls rate in an Elderly Care ward by $20 \%$ and to improve post-fall care. A baseline audit reviewed incident report forms to establish the number of falls per 1000 patient bed days for one calendar year; the baseline falls rate was 14.70 falls per 1000 bed days between November 2010 and October 2011. A care plan to highlight at-risk patients and allow adaptation of care, a 'walking-stick' incentive poster to encourage nursing staff, and post-fall guidelines, were introduced. Feedback sessions with ward staff and a re-audit were organised subsequent to each intervention. Completion of the care plan was monitored to improve compliance. A re-audit at one year was conducted to assess impact.

Feedback was positive regarding the interventions. Monthly monitoring of care plans achieved a compliance rate of $89 \%$ and highlighted up to $81 \%$ of patients were considered high-risk. The inpatient falls rate, re-audited at one year, was 12.44 falls / 1000 patient bed days, November 2011 to October 2012; a 15.4\% reduction.

This study demonstrates a $15.4 \%$ reduction in falls through use of a multi-factorial assessment and care plan and an incentive poster. As we are yet to obtain our initial goal of $20 \%$, implementation and re-audit is ongoing.

\section{Problem}

Falls are the most common patient safety incident reported to the National Patient Safety Agency (NPSA) and can lead to injury or death (1) and incur considerable cost for the NHS. (2) In 2010 the NPSA recommended NHS organisations minimise the risk of inpatient falls, though use of falls prevention teams, production of local targets for reducing harm due to falls, focused efforts on time or places falls are most likely to occur, provision of falls prevention training for staff, and detailed prevention plans for vulnerable patients. (3) We found that inpatient falls were the commonest reported incident in the Elderly Care wards; as such they are an area of significant risk regarding patient safety.

\section{Background}

Annually, approximately 282,000 inpatient falls are reported to the National Patient Safety Agency, making them the most common patient safety incident reported. Significant numbers of falls result in death or moderate to severe injury, (1) including over 1065 serious injuries and at least 83 directly attributable deaths, with costs of approximately £15 million per annum. Most inpatient falls involve patients aged over 75 years with significant co-morbidities, (2) patients typically found in Elderly Care wards.
Research shows inpatient falls may be reduced by 18 to $31 \%$ through multi-factorial assessments and interventions. (4) However, national audits have found low levels and/or poor implementation of relevant evidence-based assessments and interventions throughout the UK. (2)

The FallSafe project, (http://www.rcplondon.ac.uk/projects/fallsafe) a quality improvement initiative focusing on prevention and management of falls, identified that successful studies used ward based leaders rather than visiting specialists, engaged a multidisciplinary team, and addressed five to fifteen risk factors for falls in their interventions. In addition it was noted that combinations of evidence-based interventions were required to improve patient outcomes. (2)

It is acknowledged that not all falls can be prevented without unacceptable restrictions to patients' independence or dignity, (2) and if a fall cannot be prevented, a prompt and effective response should ensue, to achieve the best possible recovery, and avoidance of further falls.

\section{Baseline Measurement}

This project was carried out in a 19 bed unit, one of four Elderly Care wards. A baseline audit was undertaken to establish the total 
number of falls on the ward for one calendar year, by review of incident report forms. The number of patient bed days for the same year was obtained and the inpatient falls rate was calculated as the number of falls / 1000 patient bed days. The baseline falls rate was 14.70 falls / 1000 bed days for November 2010 - October 2011.

All unobserved falls, including those reported as 'found on floor' were included. Observed falls were included, except those reported as 'assisted to floor'.

\section{Design}

The aims of the project were to reduce inpatient falls by $20 \%$ and to improve quality of care provided post-fall. A ward-based medical doctor was identified as clinical lead. Using 'plan do study act' learning cycles, the clinical lead led a multi-disciplinary team to introduce care plans, incentive posters and post-fall management guidelines. Feedback sessions were organised with ward staff to review interventions and post-intervention re-audits were conducted to assess impact.

Results were disseminated via ward feedback sessions, departmental educational meetings, and wider to medical specialties through the hospital audit programme and trust-wide Safety, Quality and Effectiveness programme. The initial intervention was a multi-factorial falls assessment and care plan that has been used successfully in other falls reduction studies. (5) This form was completed by nursing staff; the first part uses NPSA's 'four screening questions' to elicit a history of falls prior to admission, after admission, anxiety about falling, and attempts to walk alone despite unsteadiness. The patient is considered at high risk of falls if a 'yes' response is obtained to any of the screening questions and a more thorough assessment and intervention is then performed, using the second part of the form. (2)

The second part of the care plan examined areas of potential increased risk for falls, such as orthostatic hypotension, bladder frequency, and medications, (2) thus enabling adaption of care to prevent falls.

The care plans were introduced initially for use with one consultant's patients who were admitted with a fall. A feedback session with ward staff was organised; problems including where to place care plans in notes to ensure completion and regular review, and how long nursing staff should have to complete them, were identified and addressed promptly to suit the working of the ward. Feedback sessions with ward staff were positive as nursing staff subjectively felt the care plans identified high-risk patients and enabled adaption of care to suit individuals' needs.

\section{Strategy}

As a result of the positive feedback, it was decided that the care plans should be completed for all patients admitted to the ward, regardless of reason for admission or consultant. Monthly monitoring of care plan completion achieved a compliance rate of $89 \%$, and highlighted that up to $81 \%$ of patients were considered high-risk. A re-audit, three months after the initial introduction of the care plans, showed a $13.4 \%$ reduction in the inpatient falls rate compared with baseline (12.73 falls / 1000 patient bed days, November 2011- January 2012).

A 'walking-stick' poster was introduced as a visual incentive to aid and encourage nursing staff. This was completed on a daily basis and highlighted if a fall occurred, allowing annotation regarding number, location, or reason for the fall.

Feedback was positive regarding use of the 'walking-stick' poster as staff stated it was a good visual reminder of progress and provided an incentive to continue.

A six month re-audit of the inpatient falls rate was conducted following the introduction of both interventions. This showed a $10.6 \%$ reduction in the inpatient falls rate $(13.14$ falls $/ 1000$ patient bed days, November 2011 to April 2012).

Trust guidelines regarding falls were published during the project. These included a post-fall assessment poster to aid medical and nursing staff in post-fall management, focusing on head, neck and pelvic/hip injuries. A feedback session with ward staff was again organised and this was positive, with subjective improvement in quality of post-fall care.

\section{Post-Measurement}

A one year re-audit was conducted to assess the on-going effects of the interventions. The inpatient falls rate had reduced by $15.4 \%$ (12.44 falls / 1000 patient bed days for November 2011 to October 2012). Yet to obtain our initial goal of a $20 \%$ reduction in the inpatient falls rate, we intend to continue implementing and monitoring the changes described, with further re-auditing as required: we need to ensure the reduction we have achieved is sustained.

We have shared the learning in this project throughout our department and intend to extend the work to the other three Elderly Care wards. We have fed back the learning to other medical specialties through local audit meetings. We also intend to hand ownership of the project to a clinical nurse lead, to maintain and improve inpatient falls rates.

\section{Lessons and Limitations}

The number of falls in an Elderly Care ward can be reduced by the use of a multi-factorial assessment and interventional care plan. A multidisciplinary team approach is necessary for success and we would suggest a dedicated time for the clinical lead to educate staff. In hindsight, as most interventions are nurse-orientated, a wardbased nurse would be best placed to fulfil the role of clinical lead.

\section{Conclusion}

Falls are the most common patient safety incident reported, frequently resulting in moderate to severe injury or death; thus 
reduction of falls and adequate post-fall management is of utmost importance. This study has demonstrated good compliance with a multi-factorial falls assessment and care plan and an incentive poster, as well as positive feedback from staff regarding usage. The inpatient falls rate decreased by $15.4 \%$ over one year: still to obtain our initial goal of a $20 \%$ reduction in the inpatient falls rate, we intend to continue implementing and monitoring the changes described, with further re-auditing as required.

\section{References}

1. NPSA. Slips trips and falls in hospital data update. NPSA 2010.

2. Darowski A, Healey F. FallSafe. Royal College of Physicians 2012.

3. NPSA. New data prompts falls prevention reminder to NHS organisations. NPSA 2010.

4. Oliver D, Healey F, Haines T. Preventing falls and fall-related injuries in hospitals. Clin Geriatr Med 2010 Nov;26(4):645-92.

5. Falls care plan - Healey F, Monro A, Cockram A, Adams V, Heseltine D. Using targeted risk factor reduction to prevent falls in older in-patients: a randomised controlled trial. Age Ageing 2004 Jul;33(4):390-5. 